\title{
Impedance Decomposition for Design-Oriented Analysis of Grid-Forming Voltage-Source Converters
}

\author{
Yicheng Liao, Student Member, IEEE, Xiongfei Wang, Senior Member, IEEE
}

\begin{abstract}
Grid-forming voltage-source converters (VSCs) tend to suffer from sideband oscillations in stiff grids. Conventional state-space eigenvalue analysis or impedance-based stability analysis based on generalized Nyquist stability criterion can only analyze how specific control parameters affect the overall system stability, which ends up with less insight into a controller designoriented analysis. To tackle this challenge, this article starts with the impedance modeling and stability analysis of grid-forming VSCs in stiff grids. Then, an impedance decomposition approach to characterizing the impacts of multiple control loops is proposed, which benefits in a controller design-oriented analysis for stability enhancement. Finally, simulation and experimental results are provided to validate the approach.
\end{abstract}

\section{INTRODUCTION}

Voltage-source converters (VSCs) become predominant in modern power systems, as the penetration of renewable energy sources increases [1]. Those VSCs are, in general, operating in the grid-following mode [2], where the VSCs feed the power to the grid via the vector current control which is synchronized with the voltage at the point of connection (PoC). However, such control schemes can lead to sideband oscillations [3] when VSCs are operating in weak grids with low short-circuit capability [3]-[5]. This phenomenon can also be termed as sub-synchronous oscillations (SSOs) if the two oscillation frequencies are within twice of the fundamental frequency [6]. The instability of grid-following VSCs in weak grids have been extensively studied [4], [7]-[10]. It is found that the phase-locked loop (PLL) used for the grid synchronization adds a negative resistance within its control bandwidth, which tends to destabilize the system in weak grids [9].

To enhance the stability of VSCs in weak grids, the gridforming control techniques are emerging in recent years as competitive alternatives [11]-[14]. Differing from the gridfollowing control, the grid-forming control regulates the VSC output voltage and achieves synchronization with the grid based on the injected power [15], such that the PLL can be removed. The recent works have proved that the grid-forming converters achieve better stability performance than the gridfollowing converters in weak grids [16]-[19]. However, the grid-forming converters may also suffer from instability with stiff grids [20]. To address the stability issue, small-signal modeling and analysis approaches have been performed on grid-forming VSCs. The existing methods for stability analysis can be classified into two categories: i) the state-space eigenvalue analysis and ii) the impedance-based analysis.

The state-space eigenvalue analysis, also named as the modal analysis, analyzes the eigenvalues of system state matrix based on the time-domain state-space model. Then, the participation factor [21] and parameter sensitivity analysis can be utilized to evaluate how state variables and control parameters affect the overall system stability [22]. It has been shown that the stability of grid-forming converters is impacted by many factors, including the droop control coefficient [23], the voltage control [21], [24], and the operating point of the system [25]. Although accurate, the state-space model characterizes the overall system dynamics, thus the analysis result only applies for a specific grid condition. The modeling and analysis need to be redone if the grid condition is changed. Therefore, the adverse effects of VSC control loops on the VSC-grid interaction explained from the VSC terminal dynamics are still unknown.

To tackle this issue, the impedance-based analysis has been adopted in the analysis of the VSC-grid interaction. In this approach, the whole system is partitioned at the $\mathrm{PoC}$ of VSC, where the VSC impedance and grid impedance are modeled separately to characterize the voltage-current relationships seen from each side [26]. Then, the system stability can be analyzed in two ways: a) the passivity-based analysis of the VSC impedance [27], b) the Nyquist stability criterion (NSC) applied to the minor loop gain formed by the VSC impedance and grid impedance.

The passivity-based analysis provides a sufficient condition for system stability, i.e., the VSC-grid interaction is stable if each subsystem is stable and passive [27]. The non-passive (or non-dissipative) behavior induced by VSC controls can destabilize VSC-grid interactions. The passivity-based analysis reported in [28] has identified the voltage feedforward loop in grid-forming VSCs can add negative damping in the sub-synchronous frequency range, which tends to destabilizes the system with stiff grids. However, this work overlooks the dynamic impacts of the outer-loop power control. Thus, the analysis is inadequate to reveal the instability mechanism of grid-forming VSCs.

The NSC-based analysis can accurately predict the system stability based on the VSC and grid impedances. Some works have applied this approach to address the stability of gridforming VSCs by using the $d q$-frame [29]-[31] or $\alpha \beta$-frame [32] impedance model. Such a model characterizes the full control dynamics into the VSC output impedance, yet its multi-input multi-output (MIMO) nature requires applying the generalized NSC for the stability analysis [33] or a simplified single-input single-output (SISO) impedance-based stability analysis considering the grid interaction [32]. The former one applies the analysis by calculating the eigenvalue transfer functions of the minor-loop-gain matrix, and the latter one relies on deriving an equivalent SISO model, both of which lose the physical meaning of the VSC impedance itself. 
Consequently, they cannot explicitly reveal how VSC control loops interact with each other, and why their interactions affect the stability. This drawback makes it difficult to develop a holistic design approach for the multi-loop control system of VSCs.

To address the challenges with the aforementioned analysis methods, this paper explores further on the stability of gridforming VSCs in stiff grids based on the impedance modeling. The presented work is discerned from the prior art in two major aspects:

a) The instability mechanism of grid-forming VSCs in stiff grids is elucidated based on the impedance singular value and passivity analysis.

b) An impedance decomposition approach is proposed to characterize the VSC control-loop impacts, which contributes to a design-oriented analysis for the gridforming VSCs.

The rest of the paper is organized as follows. Section II derives the $\alpha \beta$-frame impedance model of the droop-controlled grid-forming converter. Section III elucidates the instability mechanism of grid-forming VSCs in stiff grids based on the impedance singular value and passivity analysis. To address the stability issue, Section IV proposes an impedance decomposition method to represent the dynamic effects of multiple control loops, based on which a design-oriented analysis is conducted. Simulation and experimental validations are provided in Section V. Section VI finally concludes the paper.

\section{MODELING OF GRID-FORMING VSCS}

A three-phase grid-forming VSC is shown in Fig. 1 with a single-line representation, which is connected to a grid through the grid impedance $L_{\mathrm{g}}$. The droop control with lowpass filters (LPFs) [34], i.e., one of the typical grid-forming control schemes, is utilized for the power synchronization, and the capacitor-voltage and inductor-current dual-loop control [35] is employed for the voltage regulation. The impedance model of the VSC is derived in the $\alpha \beta$ frame, and the derivation starts from the inner voltage loop to the outer power loop. All the circuit and control parameters are listed in Tables I-II.

\section{A. Voltage Control Modeling}

First, considering the inner-loop voltage control only, the three-phase VSC system can be characterized with a SISO model in the $\alpha \beta$ frame, since the plant and control dynamics in the $\alpha$ axis and the $\beta$ axis are identical with no cross-couplings [36]. Fig. 2 shows the derived small-signal model of the dualloop voltage-controlled VSC [37]. $Z_{\mathrm{ol}}, G_{u v}, G_{i i}$, and $G_{u i}$ are the open-loop transfer functions of the plant, which are

$$
\begin{aligned}
Z_{\mathrm{ol}} & =\frac{Z_{L 1}}{1+Z_{L 1} Y_{C f}}, \\
G_{u v} & =\frac{1}{1+Z_{L 1} Y_{C f}},
\end{aligned}
$$

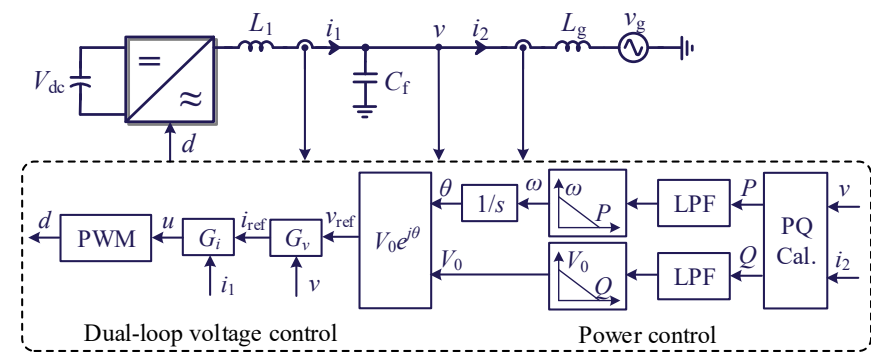

Fig. 1 Grid-connected grid-forming VSC with droop-based power control and dual-loop voltage control.

TAble I. Circuit Parameters

\begin{tabular}{c|c|c}
\hline \hline Variable & Symbol & Value \\
\hline Grid voltage (line to line) & $V_{\mathrm{g}}$ & $190 \mathrm{~V}$ \\
\hline Nominal fundamental frequency & $f_{0}\left(\omega_{0}\right)$ & $50 \mathrm{~Hz}(2 \pi \cdot 50 \mathrm{rad} / \mathrm{s})$ \\
\hline LC filter inductance & $L_{1}$ & $2 \mathrm{mH}$ \\
\hline LC filter capacitance & $C_{\mathrm{f}}$ & $10 \mu \mathrm{F}$ \\
\hline Grid inductance & $L_{\mathrm{g}}$ & $6 \mathrm{mH} / 8 \mathrm{mH}$ \\
\hline Short circuit ratio & $\mathrm{SCR}$ & $10 / 7$ \\
\hline \hline
\end{tabular}

TABle II. VSC Control Parameters

\begin{tabular}{c|c|c}
\hline \hline Variable & Symbol & Value \\
\hline Fundamental frequency & $f_{1}\left(\omega_{1}\right)$ & $50 \mathrm{~Hz}(2 \pi \cdot 50 \mathrm{rad} / \mathrm{s})$ \\
\hline Switching/sampling frequency & $f_{\mathrm{s}}$ & $10 \mathrm{kHz}$ \\
\hline LPF cut-off frequency & $f_{\mathrm{c}}\left(\omega_{\mathrm{c}}\right)$ & $1 \mathrm{~Hz}(2 \pi \cdot 1 \mathrm{rad} / \mathrm{s})$ \\
\hline Rated power & $P_{0}, Q_{0}$ & $2 \mathrm{~kW}, 2 \mathrm{kVar}$ \\
\hline Power reference & $P_{\text {ref }}, Q_{\mathrm{ref}}$ & $2 \mathrm{~kW}, 0 \mathrm{Var}$ \\
\hline Rated voltage reference & $V_{\mathrm{ref}}$ & $190 \mathrm{~V}$ \\
\hline Active power droop coefficient & $m_{P}$ & $2 \% \omega 0 / P_{0}$ \\
\hline Current controller P gain & $n_{Q}$ & $7 V_{\mathrm{ref}} / Q_{0}$ \\
\hline Voltage controller P gain & $K_{\mathrm{pi}}$ & $0.01 \mathrm{~S}$ \\
\hline Voltage controller R gain & $K_{\mathrm{rv}}$ & $50 \mathrm{~S} / \mathrm{s} / 150 \mathrm{~S} / \mathrm{s}$ \\
\hline Voltage control bandwidth & $\mathrm{BW}$ & $57 \mathrm{~Hz} / 167 \mathrm{~Hz}$ \\
\hline \hline
\end{tabular}

where $Z_{\mathrm{L} 1}$ and $Y_{\mathrm{Cf}}$ denote the impedance and admittance of the filter inductor and filter capacitor, respectively.

The current control adopts a proportional (P) controller and the voltage control adopts a proportional + resonant $(\mathrm{PR})$ controller [38]. They are implemented in the $\alpha \beta$ frame, whose transfer functions are denoted by $G_{i}$ and $G_{v}$, respectively. $G_{\mathrm{d}}$ represents the impact of the time delay [39]. The transfer functions of the controllers and the time delay are given as 


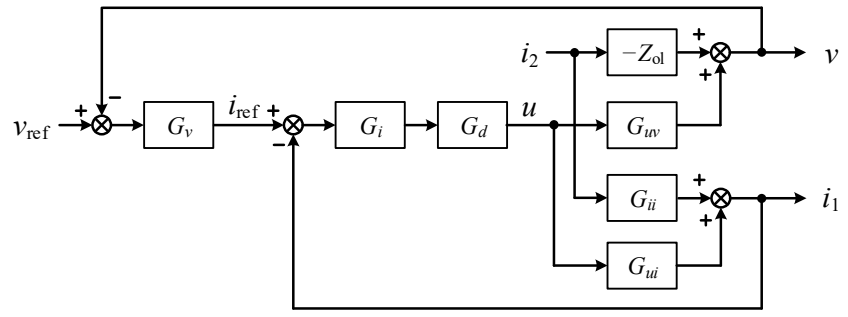

Fig. 2 Small-signal model of the inner-loop voltage control.

$$
\begin{gathered}
G_{i}=K_{\mathrm{pi}}, \\
G_{v}=K_{\mathrm{pv}}+\frac{K_{\mathrm{rv}} s}{s^{2}+\omega_{1}^{2}}, \\
G_{\mathrm{d}}=e^{-s T_{\mathrm{d}}}=e^{-1.5 s T_{\mathrm{s}}} .
\end{gathered}
$$

Then the closed-loop transfer function of the voltage loop and its output impedance can be derived from Fig. 2 as

$$
\begin{gathered}
G_{v v}=\frac{v}{v_{\mathrm{ref}}}=\frac{G_{u v} G_{d} G_{i} G_{v}}{1+G_{u i} G_{d} G_{i}+G_{u v} G_{d} G_{i} G_{v}}, \\
Z_{\mathrm{o}}=-\frac{v}{i_{2}}=\frac{Z_{\mathrm{ol}}\left(1+G_{u i} G_{d} G_{i}\right)+G_{u v} G_{d} G_{i} G_{i i}}{1+G_{u i} G_{d} G_{i}+G_{u v} G_{d} G_{i} G_{v}} .
\end{gathered}
$$

\section{B. Power Control Modeling}

To model the control dynamic of the power loop, the $\alpha \beta$ frame complex vectors are defined with $\mathbf{v}=\mathbf{v}_{\alpha}+j \mathbf{v}_{\beta}$ and $\mathbf{v}^{*}=\mathbf{v}_{\alpha}-j \mathbf{v}_{\beta}$ [10], [40]. Then, the instantaneous active power and reactive power can be calculated with the complex vectors as

$$
\begin{gathered}
P=\frac{1}{2}\left(\mathbf{v i}_{2}^{*}+\mathbf{v}^{*} \mathbf{i}_{2}\right), \\
Q=\frac{1}{2 j}\left(\mathbf{v i}_{2}^{*}-\mathbf{v}^{*} \mathbf{i}_{2}\right) .
\end{gathered}
$$

The LPFs and the droop controllers are applied to $P$ and $Q$ to obtain $\theta$ and $V_{0}$, respectively. The detailed control diagrams are given in Fig. 3, based on which the transfer functions for the $P$ and $Q$ loops can be represented by

$$
\begin{aligned}
& G_{P}(s)=-\frac{\omega_{c}}{s+\omega_{c}} \cdot \frac{m_{P}}{s}, \\
& G_{Q}(s)=-\frac{\omega_{c}}{s+\omega_{c}} \cdot n_{Q} . \\
& {\left[\begin{array}{c}
\mathbf{v}_{\mathrm{ref}} \\
e^{j 2 \theta_{1}} \mathbf{v}_{\mathrm{ref}}^{*}
\end{array}\right]=-\underbrace{-\frac{j}{2}\left[\begin{array}{cc}
-\left(V_{0} G_{P}\left(p-j \omega_{1}\right)-G_{Q}\left(p-j \omega_{1}\right)\right) I_{2 d q}^{*} & -\left(V_{0} G_{P}\left(p-j \omega_{1}\right)+G_{Q}\left(p-j \omega_{1}\right)\right) I_{2 d q} \\
\left(V_{0} G_{P}\left(p-j \omega_{1}\right)+G_{Q}\left(p-j \omega_{1}\right)\right) I_{2 d q}^{*} & \left(V_{0} G_{P}\left(p-j \omega_{1}\right)-G_{Q}\left(p-j \omega_{1}\right)\right) I_{2 d q}
\end{array}\right]}_{\mathbf{G}_{\mathrm{ref}}}\left[\begin{array}{c}
\mathbf{v} \\
e^{j 2 \theta_{1}} \mathbf{v}^{*}
\end{array}\right]} \\
& \underbrace{-\frac{j}{2}\left[\begin{array}{cc}
-\left(V_{0} G_{P}\left(p-j \omega_{1}\right)+G_{Q}\left(p-j \omega_{1}\right)\right) V_{d q}^{*} & -\left(V_{0} G_{P}\left(p-j \omega_{1}\right)-G_{Q}\left(p-j \omega_{1}\right)\right) V_{d q} \\
\left(V_{0} G_{P}\left(p-j \omega_{1}\right)-G_{Q}\left(p-j \omega_{1}\right)\right) V_{d q}^{*} & \left(V_{0} G_{P}\left(p-j \omega_{1}\right)+G_{Q}\left(p-j \omega_{1}\right)\right) V_{d q}
\end{array}\right]}_{\mathbf{Z}_{\mathrm{ref}}}\left[\begin{array}{c}
\mathbf{i}_{2} \\
e^{j 2 \theta_{1}} \mathbf{i}_{2}^{*}
\end{array}\right]
\end{aligned}
$$
only to $\mathbf{v}$ and $\mathbf{i}_{2}$, but also to $e^{j 2 \theta_{1}} \mathbf{v}^{*}$ and $e^{j 2 \theta_{1}} \mathbf{i}_{2}^{*}$.
Then, the $\alpha \beta$-frame voltage reference is generated as

$$
\mathbf{v}_{\text {ref }}=V_{0} e^{j \theta},
$$

whose linearized small-signal model can be derived as

$$
\hat{\mathbf{v}}_{\text {ref }}=j V_{0} e^{j \theta_{1}} \hat{\theta}+e^{j \theta_{1}} \hat{V}_{0}=j V_{0} e^{j \theta_{1}} G_{P}(p) \hat{P}+e^{j \theta_{1}} G_{Q}(p) \hat{Q},
$$

where $G_{P}(p)$ and $G_{Q}(p)$ denote the time-domain operators of the transfer functions $G_{P}(s)$ and $G_{Q}(s)$ with $p=\mathrm{d} / \mathrm{d} t$. $\theta_{1}$ denotes the time-variant phase of the VSC output voltage at the fundamental frequency.

It is seen from (10) and (11) that $P$ and $Q$ are calculated by the nonlinear equations that consist of the multiplications of voltages and currents, and thus the linearization should be also done when substituting (10) and (11) into (15), yielding

$$
\begin{aligned}
\hat{\mathbf{v}}_{\text {ref }}= & \frac{j}{2} V_{0} e^{j \theta_{1}} G_{P}(p)\left(I_{2 d q}^{*} e^{-j \theta_{1}} \hat{\mathbf{v}}+V_{d q} e^{j \theta_{1}} \hat{\mathbf{i}}_{2}^{*}+V_{d q}^{*} e^{-j \theta_{1}} \hat{\mathbf{i}}_{2}+I_{2 d q} e^{j \theta_{1}} \hat{\mathbf{v}}^{*}\right) \\
& -\frac{j}{2} e^{j \theta_{1}} G_{Q}(p)\left(I_{2 d q}^{*} e^{-j \theta_{1}} \hat{\mathbf{v}}+V_{d q} e^{j \theta_{1}} \hat{\mathbf{i}}_{2}^{*}-V_{d q}^{*} e^{-j \theta_{1}} \hat{\mathbf{i}}_{2}-I_{2 d q} e^{j \theta_{1}} \hat{\mathbf{v}}^{*}\right) \\
= & \frac{j}{2} V_{0} G_{P}\left(p-\omega_{1}\right)\left(I_{2 d q}^{*} \hat{\mathbf{v}}+V_{d q} e^{j 2 \theta_{1}} \hat{\mathbf{i}}_{2}^{*}+V_{d q}^{*} \hat{\mathbf{i}}_{2}+I_{2 d q} e^{j 2 \theta_{1}} \hat{\mathbf{v}}^{*}\right) \\
& -\frac{j}{2} G_{Q}\left(p-\omega_{1}\right)\left(I_{2 d q}^{*} \hat{\mathbf{v}}+V_{d q} e^{j 2 \theta_{1}} \hat{\mathbf{i}}_{2}^{*}-V_{d q}^{*} \hat{\mathbf{i}}_{2}-I_{2 d q} e^{j 2 \theta_{1}} \hat{\mathbf{v}}^{*}\right)
\end{aligned}
$$

where $V_{d q}$ and $I_{2 d q}$ are defined as the steady-state values of the VSC output voltage and current in the synchronous reference frame of $\theta_{1}$. It is noted that the dynamic of $\mathbf{v}_{\text {ref }}$ is related not

To derive a unified form for the ac voltage and current variables, the complex conjugate and the angle rotation of $e^{j 2 \theta_{1}}$ are applied successively to (16), which yields

$$
\begin{aligned}
e^{j 2 \theta_{1}} \hat{\mathbf{v}}_{\text {ref }}^{*}= & -\frac{j}{2} V_{0} G_{P}\left(p-\omega_{1}\right)\left(I_{2 d q} e^{j 2 \theta_{1}} \hat{\mathbf{v}}^{*}+V_{d q}^{*} \hat{\mathbf{i}}_{2}+V_{d q} e^{j 2 \theta_{1}} \hat{\mathbf{i}}_{2}^{*}+I_{2 d q}^{*} \hat{\mathbf{v}}\right) \\
& +\frac{j}{2} G_{Q}\left(p-\omega_{1}\right)\left(I_{2 d q} e^{j 2 \theta_{1}} \hat{\mathbf{v}}^{*}+V_{d q}^{*} \hat{\mathbf{i}}_{2}-V_{d q} e^{j 2 \theta_{1}} \hat{\mathbf{i}}_{2}^{*}-I_{2 d q}^{*} \hat{\mathbf{v}}\right)
\end{aligned}
$$

Combining (16) and (17), the dynamic relationship from the VSC output voltage and current to the voltage reference can be derived as (18), which is given at the bottom of this page. Then the two complex transfer matrices, i.e. $\mathbf{Z}_{\text {ref }}(s)$ and $\mathbf{G}_{\text {ref }}(s)$, can be defined accordingly with $p$ replaced by $s$.

It is seen from (18) that, in the $\alpha \beta$ frame, all the ac variables should be denoted by two complex vectors. Consequently, the dynamic of the power synchronization loop is characterized by a MIMO model. The existence of off-diagonal elements in $\mathbf{Z}_{\text {ref }}$ and $\mathbf{G}_{\text {ref }}$ implies the frequency-coupling effects introduced by 

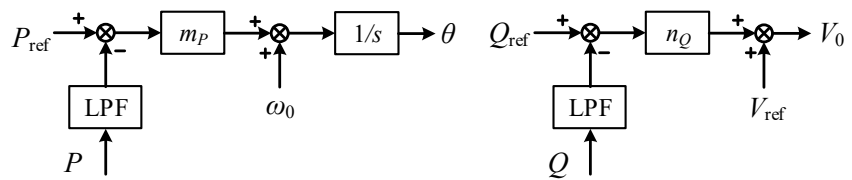

Fig. 3 Control diagram of the droop control with LPFs.

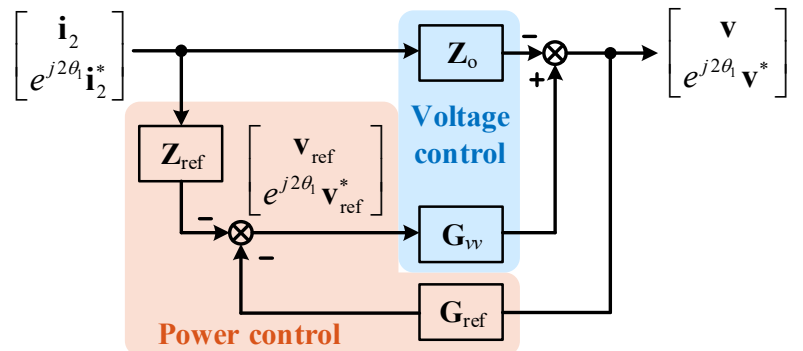

Fig. 4 Closed-loop model of VSC considering the voltage-loop and power-loop controls.

\section{VSC Impedance Model}

To integrate the inner voltage control loop into the power control loop, the SISO models of (8) and (9) are extended into the MIMO forms in the complex space, which leads to

$$
\begin{aligned}
\mathbf{G}_{v v} & =\left[\begin{array}{cc}
G_{v v}(s) & 0 \\
0 & G_{v v}\left(s-j 2 \omega_{1}\right)
\end{array}\right], \\
\mathbf{Z}_{\mathrm{o}} & =\left[\begin{array}{cc}
Z_{\mathrm{o}}(s) & 0 \\
0 & Z_{\mathrm{o}}\left(s-j 2 \omega_{1}\right)
\end{array}\right] .
\end{aligned}
$$

Since the inner-loop voltage control is linear time-invariant, there are no frequency coupling terms in the off-diagonal elements in (19) and (20). Then, the full model of the VSC can be depicted by Fig. 4, where $\mathbf{Z}_{o}$ and $\mathbf{G}_{v v}$ represent the dynamics of the inner-loop voltage control, $\mathbf{Z}_{\text {ref }}$ and $\mathbf{G}_{\text {ref }}$ represent the dynamics of the outer-loop power control. The output impedance of the VSC is then derived as

$$
\begin{aligned}
& {\left[\begin{array}{c}
\mathbf{v} \\
e^{j 2 \theta_{1}} \mathbf{v}^{*}
\end{array}\right]=-\mathbf{Z}_{\mathrm{VSC}}\left[\begin{array}{c}
\mathbf{i}_{2} \\
e^{j 2 \theta_{1}} \mathbf{i}_{2}^{*}
\end{array}\right] \text { where }} \\
& \mathbf{Z}_{\mathrm{vSC}}=\left(\mathbf{I}+\mathbf{G}_{\mathrm{vv}} \mathbf{G}_{\mathrm{ref}}\right)^{-1}\left(\mathbf{Z}_{\mathrm{o}}+\mathbf{G}_{\mathrm{vv}} \mathbf{Z}_{\mathrm{ref}}\right) \triangleq\left[\begin{array}{ll}
Z_{\mathrm{VSC} 11} & Z_{\mathrm{VSC} 12} \\
Z_{\mathrm{VSC} 21} & Z_{\mathrm{VSC} 22}
\end{array}\right]
\end{aligned}
$$

$\mathbf{Z}_{\mathrm{VSC}}$ is a MIMO model represented in the $\alpha \beta$ frame. The four elements of (21) are defined as $Z_{\mathrm{VSC} 11}, Z_{\mathrm{VSC} 12}, Z_{\mathrm{VSC} 21}$, and $Z_{\mathrm{VSC} 22}$.

\section{Impedance Model Validation}

One of the advantages of the impedance-based analysis over the state-space analysis is that the VSC can be treated as a "black-box" system, and its impedance can be obtained from frequency scanning. Perturbing the VSC with frequencyscanned small signals around its operating point/ trajectory, the frequency response of the linearized small-signal impedance model of the VSC can be calculated, by applying the discrete Fourier transformation (DFT) analysis of the measured voltage and current waveforms at the PoC [41].

Using the impedance measurement method proposed in [40], the derived $\alpha \beta$-frame impedance model is validated via
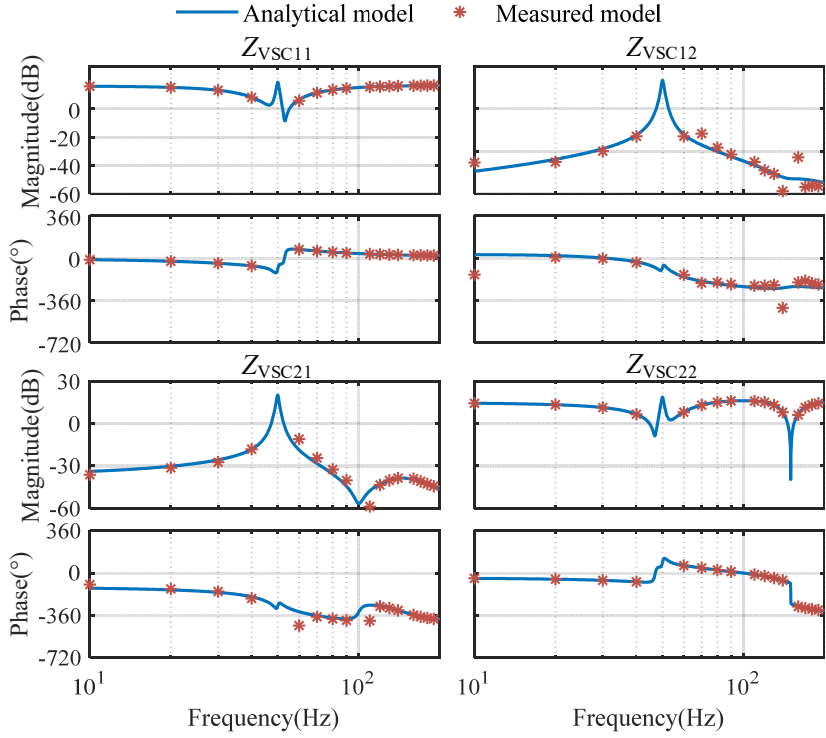

Fig. 5 Measure impedance model compared with the analytical model of the grid-forming VSC.

switching models in MATLAB/Simulink. Since the frequency coupling behavior is introduced by the power control dynamics, which is strongest around the fundamental frequency, the frequency is scanned within $(0 \mathrm{~Hz}, 200 \mathrm{~Hz})$. The measured impedance is plotted by asterisks shown in Fig. 5 , which shows in good agreement with the analytical model. It verifies the accuracy of the VSC modeling.

\section{INSTABILITY MECHANISM IN STIFF GRIDS}

This section formulates the problem by discussing the instability mechanism of the grid-forming VSC in stiff grids.

\section{A. Combined Passivity and Small-Gain Stability Criterion}

The combined small-gain and passivity stability criterion [42] is introduced first:

Criterion 1: For an interconnected system formed by the return ratio of $\mathbf{L}(s)=\mathbf{Q}(s) \mathbf{P}(s)$, if $\mathbf{P}(s)$ is strictly passive, and $\mathbf{Q}(s)$ is stable and passive in specific frequency bands, the closed-loop system is stable if $\bar{\sigma}[\mathbf{Q}(j \omega)] \bar{\sigma}[\mathbf{P}(j \omega)]<1$ in the non-passive frequency bands of $\mathbf{Q}(s)$, where $\bar{\sigma}$ means the maximum singular value.

It is noted that Criterion 1 is merely a sufficient condition for stability, which may not be readily met in practical stable systems. However, it is inspired that the stability analysis with the generalized NSC can be simply focused in the non-passive frequency bands of $\mathbf{Q}(s)$, which is carried out in Part B. Furthermore, the small-gain condition based on the singular value analysis can be used to clarify the instability mechanism of grid-forming VSCs in stiff grids, which is conducted in Part C.

\section{B. Stability Analysis based on Generalized NSC and Passivity}

For the VSC-grid interconnected system, the system return ratio is defined as 


$$
\mathbf{L}=\mathbf{Z}_{\mathrm{VSC}} \mathbf{Z}_{\mathrm{g}}^{-1}
$$

where $\mathbf{Z}_{\mathrm{g}}$ representing the grid impedance can be similarly derived in a MIMO form according to (20).

In such a system, $\mathbf{Z}_{\mathrm{g}}$ is strictly passive, while $\mathbf{Z}_{\mathrm{VSC}}$ may be non-passive in specific frequency bands. The passivity of $\mathbf{Z}_{\mathrm{VSC}}$ can be checked by the positive realness of the passivity index [43], which is the minimum eigenvalue of the Hermitian matrix of $\mathbf{Z}_{\mathrm{VSC}}$, i.e.,

$$
p_{\min }(\omega)=\min \lambda\left\{\frac{1}{2}\left(\mathbf{Z}_{\mathrm{vSC}}(j \omega)+\mathbf{Z}_{\mathrm{vSC}}^{\mathrm{H}}(j \omega)\right)\right\} .
$$

Based on the generalized NSC [44], the system is stable if and only if the two eigenvalue transfer functions of $\mathbf{L}$ satisfy the NSC. Then, the VSC-grid system stability can be checked by combining the Bode diagrams of the eigenvalue transfer functions of $\mathbf{L}$ and the passivity index of $\mathbf{Z}_{\mathrm{VSC}}$.

A critically unstable case (Case A) is studied first. The VSC parameters listed in Table II with the voltage control bandwidth of $57 \mathrm{~Hz}$ are utilized, and the grid impedance with the short-circuit ratio (SCR) of 10 is considered, i.e., $L_{\mathrm{g}}=6$ $\mathrm{mH}$. The steady-state operating points of the droop-controlled VSC are given as follows.

- $\mathrm{SCR}=10: I_{d}=10.48 \mathrm{~A}, I_{q}=0.22 \mathrm{~A}, V_{d}=190.94 \mathrm{~V}, V_{q}=0 \mathrm{~V}$.

Fig. 6 plots the Bode diagrams of the eigenvalue transfer functions of $\mathbf{L}$ and the passivity index of $\mathbf{Z}_{\mathrm{VSC}}$. From Fig. 6(a), there are three non-passive frequency bands, around $-50 \mathrm{~Hz}$, $50 \mathrm{~Hz}$ and $150 \mathrm{~Hz}$, respectively. It can be seen that in the two non-passive frequency bands around $-50 \mathrm{~Hz}$ and $50 \mathrm{~Hz}, \lambda_{1}$ and $\lambda_{2}$ do not cross over $\pm 180^{\circ}$, thus the frequency responses are not important to stability. Therefore, the stability analysis can be focused in the non-passive frequency band around the fundamental frequency, which is shown in the zoomed in plot in Fig. 6(b).

The NSC can be then applied to Fig. 6(b). From the Bode diagram of $\lambda_{1}$, there is a right-half-plane (RHP) pole identified at $50 \mathrm{~Hz}$, since the phase steps up at the resonant peak [45]. This RHP pole can be also observed from Fig. 5 in each impedance element, which is induced by the $\left(\mathbf{I}+\mathbf{G}_{\mathrm{vV}} \mathbf{G}_{\mathrm{ref}}\right)^{-1}$ in (21). It is noted that the existence of RHP poles does not violate the fact that the grid-forming VSC should be opencircuit stable, since in the open-circuit case, $I_{2 d q}=0$, such that the $\left(\mathbf{I}+\mathbf{G}_{\mathrm{vv}} \mathbf{G}_{\mathrm{ref}}\right)^{-1}=\mathbf{I}$. It is thus implied that the existence of RHP poles in the grid-forming VSC impedance is dependent on the VSC operating points. From the phase response of $\lambda_{1}$, in the non-passive frequency band, there are two clockwise crossings (CCs) and one anti-clockwise crossing (ACC) over $180^{\circ}$, as denoted by the red dots. The ACC compensates the impact of the RHP pole, thus does not affect the stability. For the two CCs, it can be found that the corresponding magnitude responses are both critically larger than $0 \mathrm{~dB}$, which indicates a critically unstable system.

It is worth noting that the non-passive frequency band for grid-forming VSCs is not wide around the fundamental frequency, which can limit the variation range of the potential oscillation modes. Consequently, the sideband oscillations induced by the grid-forming VSC-grid interaction are SSOs [6].

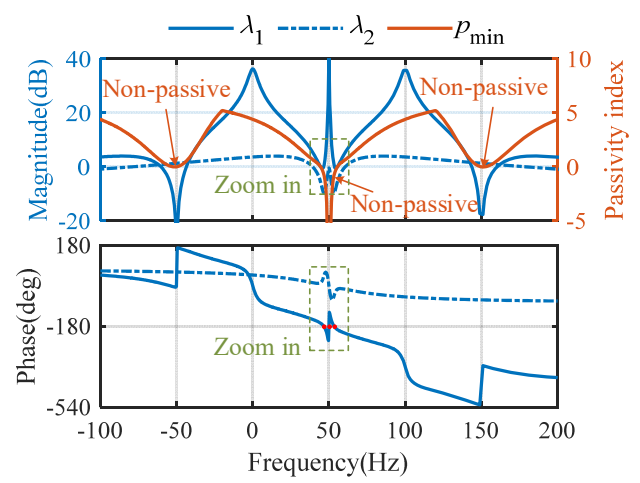

(a)

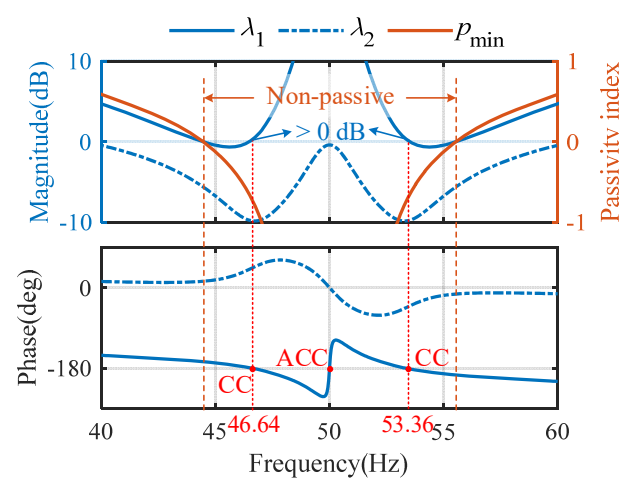

(b)

Fig. 6 Bode diagrams of eigenvalue transfer functions of $\mathbf{L}$ and passivity index of $\mathbf{Z}_{\mathrm{VSC}}$ for Case A. (a) Frequency response in wider frequency range; (b) Zoomed in plot.

\section{Stability Affected by Short Circuit Ratio}

To illustrate the impact of SCR on the stability, Case B considering a grid impedance of $L_{\mathrm{g}}=8 \mathrm{mH}(\mathrm{SCR}=7)$ is studied. The steady-state operating points of the VSC under this grid condition are given as follows.

- $\mathrm{SCR}=7: I_{d}=10.5 \mathrm{~A}, I_{q}=0 \mathrm{~A}, V_{d}=190.54 \mathrm{~V}, V_{q}=0 \mathrm{~V}$.

The stability analysis based on the generalized NSC and the passivity analysis is provided in Fig. 7, where only zoomed in plot around the fundamental frequency is shown for brevity. It can be seen that the phase crossover frequencies at the CCs are almost unchanged compared with Fig. 6(b), yet the corresponding magnitude response of $\lambda_{1}$ is less than $0 \mathrm{~dB}$. It is indicated that when the grid is weaker (SCR reduced), the system stability is improved.

However, the analysis based on the eigenvalue transfer functions of $\mathbf{L}$ cannot elucidate much insight into the interaction of the VSC and grid, which makes it difficult to understand the instability mechanism. Therefore, the singular value analysis is further conducted, which is inspired by Criterion 1. Based on the definition of $\mathbf{L}$, it can be derived that

$$
\left|\lambda_{i}(\mathbf{L})\right| \leq \bar{\sigma}\left(\mathbf{Z}_{\mathrm{VSC}}\right) \bar{\sigma}\left(\mathbf{Z}_{\mathrm{g}}^{-1}\right)=\bar{\sigma}\left(\mathbf{Z}_{\mathrm{VSC}}\right) / \underline{\sigma}\left(\mathbf{Z}_{\mathrm{g}}\right) .
$$

Then, according to the generalized NSC, the stability of the VSC-grid interaction can be ensured if $\bar{\sigma}\left(\mathbf{Z}_{\mathrm{VSC}}\right) / \underline{\sigma}\left(\mathbf{Z}_{\mathrm{g}}\right)<1$ 


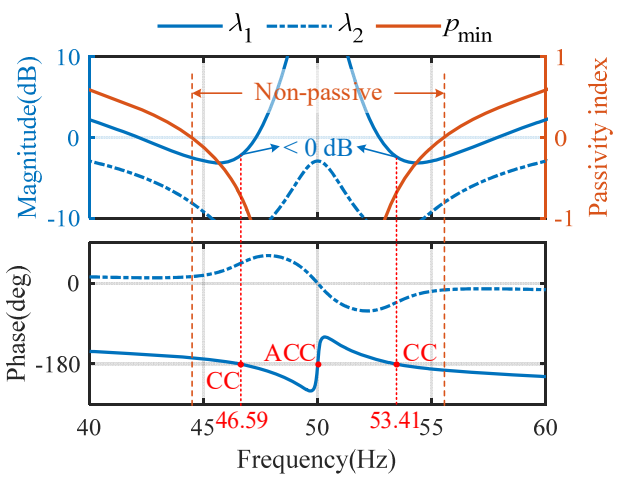

Fig. 7 Bode diagrams of eigenvalue transfer functions of $\mathbf{L}$ and passivity index of $\mathbf{Z}_{\mathrm{vsC}}$ for Case B.

is satisfied at the phase crossover frequencies in the nonpassive frequency bands of $\mathbf{Z}_{\mathrm{VSC}}$.

Fig. 8 shows the singular value analysis of $\mathbf{Z}_{\mathrm{VSC}}$ and $\mathbf{Z}_{\mathrm{g}}$ compared with the passivity index of $\mathbf{Z}_{\mathrm{VSC}}$. Since under Cases A-B with SCR changing, the operating points of the VSC are almost unchanged, indicating that the profile of $\mathbf{Z}_{\mathrm{VSC}}$ is almost unchanged. And the phase response of $\mathbf{Z}_{\mathrm{g}}$ is mainly inductive around the fundamental frequency, thus the phase crossover frequencies within the non-passive frequency band of $\mathbf{Z}_{\mathrm{VSC}}$ are almost unchanged, which are simply marked by the red dashed lines at $46.6 \mathrm{~Hz}$ and $53.4 \mathrm{~Hz}$. The singular value is an indicator of the matrix magnitude. It can be seen that the VSC impedance features high magnitude around the fundamental frequency, which tends to intersect with the grid impedance and violate the small-gain condition in Criterion 1. As the SCR increases, i.e., the grid is stiffer, the frequency band for which $\bar{\sigma}\left(\mathbf{Z}_{\mathrm{vsC}}\right)>\underline{\sigma}\left(\mathbf{Z}_{\mathrm{g}}\right)$ is widen, which tends to reduce the system gain margin at the phase crossover frequencies. This explains why the grid-forming VSC may suffer from instability in stiff grids.

However, the singular value analysis still fails in analyzing the impacts of VSC control loops, since the VSC impedance is calculated as a whole. This challenge will be addressed in next section.

\section{IMPEDANCE DECOMPOSITION FOR DESIGN-ORIENTED ANALYSIS}

This section further investigates the control-loop impacts on the stability of grid-forming VSCs, by proposing an impedance decomposition approach, which enables a designoriented analysis.

\section{A. Control-Loop-Based Impedance Decomposition}

The closed-loop VSC impedance model in Fig. 4 consists of two feedforward loops and one feedback loop. For the feedforward loops, one is characterized by $\mathbf{Z}_{\mathrm{o}}$ representing the voltage control dynamics, and the other is characterized by $\mathbf{Z}_{\mathrm{ref}} \mathbf{G}_{v v}$ mainly impacted by the power control dynamics around the fundamental frequency. According to the expression of $\mathbf{Z}_{\text {ref }}$ in (18), the contributions of the active power

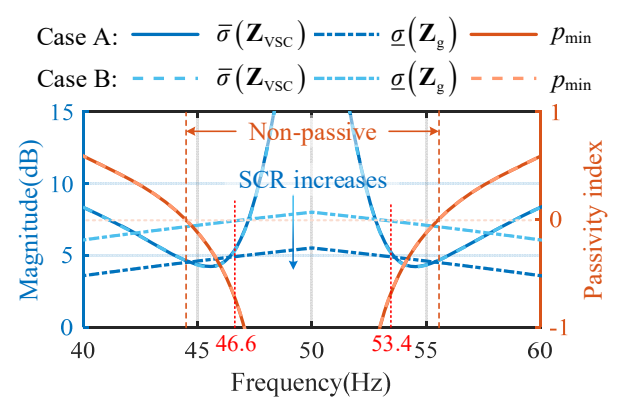

Fig. 8 Impedance singular value and passivity analysis comparing Cases A-B.

loop and the reactive power loop can be separated into $\mathbf{Z}_{\text {ref,P }}$ and $\mathbf{Z}_{\text {ref,Q }}$, respectively, i.e.,

$$
\begin{gathered}
\mathbf{Z}_{\text {refP }}=\frac{j}{2}\left[\begin{array}{cc}
-V_{0} G_{P}\left(s-j \omega_{1}\right) V_{d q}^{*} & -V_{0} G_{P}\left(s-j \omega_{1}\right) V_{d q} \\
V_{0} G_{P}\left(s-j \omega_{1}\right) V_{d q}^{*} & V_{0} G_{P}\left(s-j \omega_{1}\right) V_{d q}
\end{array}\right], \\
\mathbf{Z}_{\text {refQ }}=\frac{j}{2}\left[\begin{array}{ll}
-G_{Q}\left(s-j \omega_{1}\right) V_{d q}^{*} & G_{Q}\left(s-j \omega_{1}\right) V_{d q} \\
-G_{Q}\left(s-j \omega_{1}\right) V_{d q}^{*} & G_{Q}\left(s-j \omega_{1}\right) V_{d q}
\end{array}\right] .
\end{gathered}
$$

Then, Fig. 4 is reformulated as Fig. 9(a), where three feedforward loops are used to represent the three control loop dynamics, respectively. For abbreviation, VC represents the inner-loop voltage control, APC represents the active power control, and RPC represents the reactive power control. Consequently, the VSC impedance shown in (21) can be decomposed into several impedances in series, i.e.,

$$
\begin{aligned}
\mathbf{Z}_{\mathrm{vSC}}= & \underbrace{\left(\mathbf{I}+\mathbf{G}_{\mathrm{vv}} \mathbf{G}_{\mathrm{ref}}\right)^{-1} \mathbf{Z}_{\mathrm{o}}}_{\mathbf{Z}_{\mathrm{vC}}}+\underbrace{\left(\mathbf{I}+\mathbf{G}_{\mathrm{vv}} \mathbf{G}_{\mathrm{ref}}\right)^{-1} \mathbf{G}_{\mathrm{vv}} \mathbf{Z}_{\mathrm{refP}}}_{\mathbf{Z}_{\mathrm{APC}}} \\
& +\underbrace{\left(\mathbf{I}+\mathbf{G}_{\mathrm{vv}} \mathbf{G}_{\mathrm{ref}}\right)^{-1} \mathbf{G}_{\mathrm{vv}} \mathbf{Z}_{\mathrm{refl}}}_{\mathbf{Z}_{\mathrm{RPC}}}
\end{aligned}
$$

where each impedance represents the dynamic of each feedforward loop. Then, the equivalent circuit of the gridforming VSC by decomposing different control loops is depicted as Fig. 9(b).

It should be noted that the above control impedance decomposition is merely based on the feedforward loop separation. These control loops are still coupled with each other through the feedback loop, which results in a common factor in each decomposed impedance, i.e., $\left(\mathbf{I}+\mathbf{G}_{v v} \mathbf{G}_{\mathrm{ref}}\right)^{-1}$.

\section{B. Control-Loop Impact Analysis}

The singular value analysis in Section III-C has indicated that the impedance magnitude is critical to the stability. However, the grid-forming VSC impedance features high magnitude around the fundamental frequency, which disagrees with the common conception that a voltage-controlled VSC should have a low output impedance within its control bandwidth. The reason can be explained by analyzing the control-loop impacts based on the decomposed impedances.

According to (27), the decomposed impedances differ from each other in the feedforward loops. Therefore, the different impacts of control loops on the VSC impedance shaping can be discussed by comparing the magnitude responses of $\mathbf{Z}_{0}$, $\mathbf{Z}_{\text {reff }} \mathbf{G}_{v v}$ and $\mathbf{Z}_{\text {refe }} \mathbf{G}_{v v}$. Before comparing them together, the 


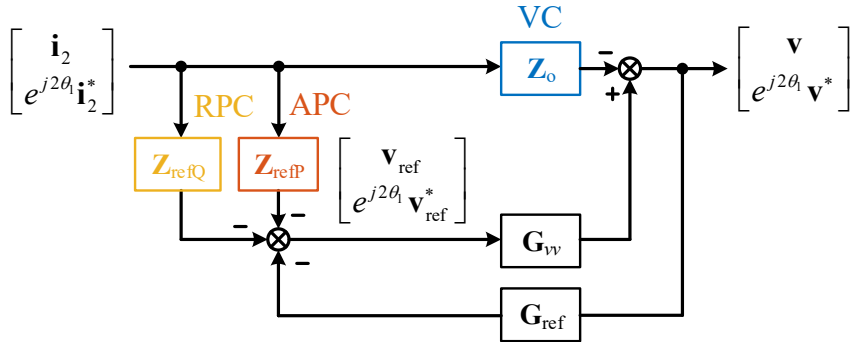

(a)

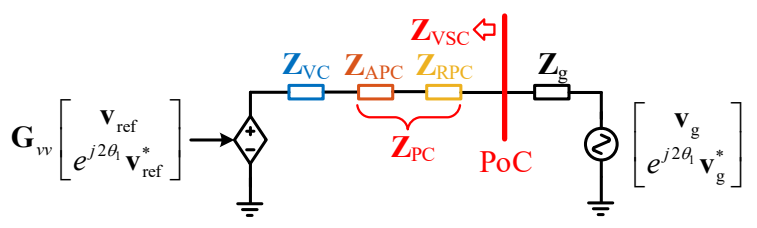

(b)

Fig. 9 Impedance decomposition approach for the grid-forming VSC. (a) Feedforward loop-based decomposition; (b) Equivalent circuit with decomposed impedances.

voltage control impact on $\mathbf{Z}_{\mathrm{o}}$ and the power control impact on $\mathbf{Z}_{\text {refP }}$ and $\mathbf{Z}_{\text {refQ }}$ are discussed first.

\section{1) Voltage Control Impact}

$\mathbf{Z}_{\mathrm{o}}$ represents the voltage control impact, which is a diagonal matrix. The second diagonal element is merely frequency shifted from the first diagonal element, thus the voltage control impact on the output impedance shaping can be clarified by the SISO model given in (9).

Since the VC output impedance is influenced by the voltage control design, the VC open-loop gain calculated by (28) and the VC output impedance are both plotted in Fig. 10, considering the two sets of VC parameters listed in Table II. The VC control bandwidth (BW) is defined based on magnitude crossover frequencies.

$$
T_{v}=\frac{G_{u v} G_{\mathrm{d}} G_{i} G_{v}}{1+G_{u i} G_{d} G_{i}} .
$$

In Fig. 10, for the VC loop gain, it can be seen that due to the impact of the LC resonance, the bandwidth cannot be designed sufficiently high [46]. $T_{v 1}$ achieves a gain margin (GM) of $6 \mathrm{~dB}$, with only a limited bandwidth of $57 \mathrm{~Hz} . T_{v 2}$ increases the bandwidth to $167 \mathrm{~Hz}$, yet sacrifices the GM to $4.8 \mathrm{~dB}$.

For the VC output impedance, its magnitude is low merely in a narrow frequency band around $f_{1}=50 \mathrm{~Hz}$. The width of this frequency band is in positive correlation to the $\mathrm{VC}$ bandwidth. In the very low frequency range approaching to 0 $\mathrm{Hz}$, the magnitude response of $Z_{\mathrm{o}}$ approaches to

$$
\lim _{\omega \rightarrow 0}\left|Z_{\mathrm{o}}(\omega)\right|=\left|\frac{K_{\mathrm{pi}}}{1+K_{\mathrm{pi}} K_{\mathrm{pv}}}\right|,
$$

which behaves as a virtual resistance emulated by the innerloop voltage and current controllers. Following the dual-loop voltage control design guideline [46], $K_{\mathrm{pv}}$ is usually designed much small to reduce the impact of the LC resonance on the VC loop gain design, such that $K_{\mathrm{pi}} K_{\mathrm{pv}}<<1$ is satisfied. Thus,

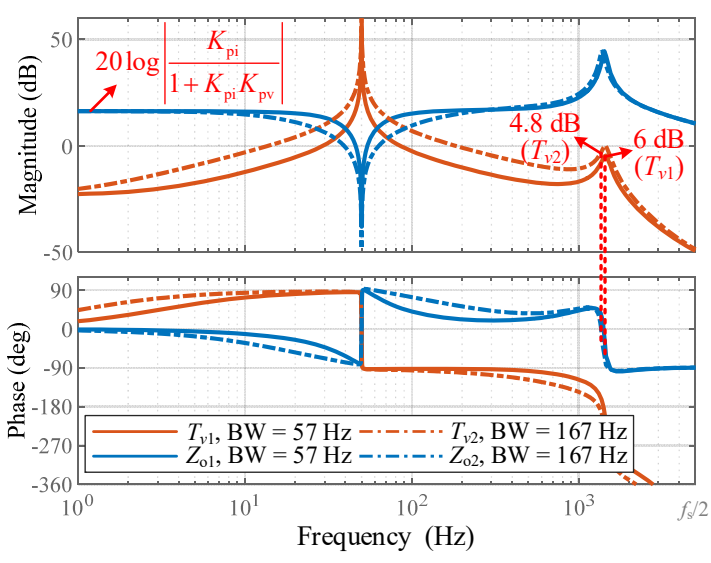

Fig. 10 Bode diagrams of VC loop gains and VC output impedances

the low-frequency (much lower than $f_{1}$ ) impedance is mainly shaped by the current controller gain, which is usually designed as large as possible, since the current controller gain is aimed to provide active damping for the LC resonance [38]. In the high-frequency range (much higher than $f_{1}$ ), it can be found that the impedance is mainly shaped by the LC resonance including the time-delay effects [37]. Consequently, the limited $\mathrm{VC}$ bandwidth results in a non-negligible $Z_{\mathrm{o}}$ around $f_{1}$, which can interact with the power control impedances and further affect the VSC output impedance.

\section{2) Power Control Impact}

The power control impact is characterized by the impedance

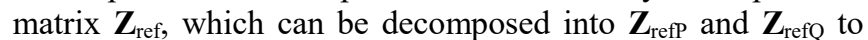
quantify the interaction of APC and RPC, respectively. Considering $V_{d q}=V_{0}$ in steady state, it can be seen that the interaction between APC and RPC can be analyzed by comparing the frequency responses of $V_{0} G_{P}\left(s-j \omega_{1}\right)$ and $G_{Q}\left(s-j \omega_{1}\right)$ according to (25), (26). Assuming the same LPF cut-off frequencies in APC and RPC, the ratio of their magnitude responses can be derived as

$$
\left|\frac{V_{0} G_{P}\left(j \omega-j \omega_{1}\right)}{G_{Q}\left(j \omega-j \omega_{1}\right)}\right|=\left|\frac{V_{0} \cdot \frac{\omega_{c}}{j \omega+\omega_{c}} \cdot \frac{m_{P}}{j \omega}}{\frac{\omega_{c}}{j \omega+\omega_{c}} \cdot n_{Q}}\right|=\frac{m_{P} V_{0}}{\omega n_{Q}}=\frac{r_{P} \omega_{0}}{r_{Q} \omega}
$$

where $r_{P}$ and $r_{Q}$ are the allowed maximum percentages of the frequency deviation and voltage deviation. Consequently, the magnitude crossover frequency of (30) can be found as $f=$ $f_{1} \pm r_{P} \cdot f_{0} / r_{Q}$. It is implied that, when $\left|f-f_{1}\right|<r_{P} \cdot f_{0} / r_{Q}$, the APC impact is stronger, and a large gain is introduced as $f$ approaches to $f_{1}$ due to the integrator used in APC. When $\mid f-$ $f_{1} \mid>r_{P} \cdot f_{0} / r_{Q}$, the RPC impact is stronger.

\section{3) Control-Loop Impacts on VSC Impedance}

To further compare the control-loop impacts on VSC impedance, the Bode diagrams of $\mathbf{Z}_{0}, \mathbf{Z}_{\text {refP }} \mathbf{G}_{v v}$ and $\mathbf{Z}_{\mathrm{refQ}} \mathbf{G}_{v v}$ are plotted together in Fig. 11. According to the magnitude analysis, it can be seen that when $f$ goes farther from the fundamental frequency, the VC's impact is much stronger than other loops, indicating $\mathrm{VC}$ is dominant. When $f$ is approaching to the fundamental frequency, the APC's impact is mostly 


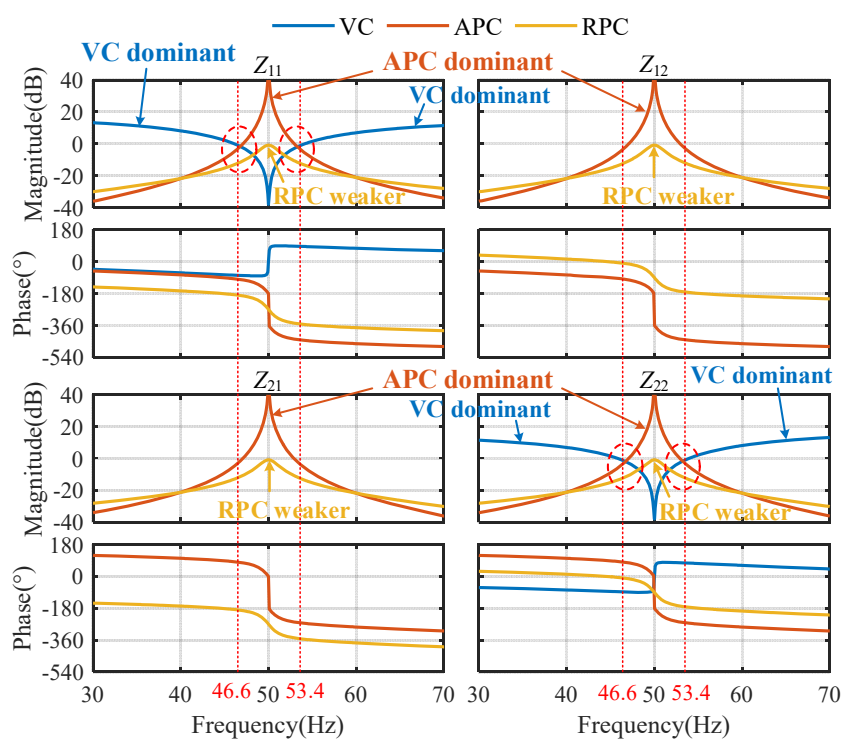

Fig. 11 Bode diagrams of $\mathbf{Z}_{\mathrm{o}}, \mathbf{Z}_{\text {refr }} \mathbf{G}_{v}$, and $\mathbf{Z}_{\text {ref }} \mathbf{G}_{v v}$.

significant, indicating the APC is dominant. The RPC's impact is relatively weaker in contrast to either the $\mathrm{VC}$ or the APC. There are some frequency bands where the VC and APC impedances intersect with each other, denoted in the red dashed ellipses. In such frequency bands, the VC and APC can have comparative impacts on the VSC impedance. According to the comparison in Fig. 11, it is learned that the high magnitude of the grid-forming VSC impedance around the fundamental frequency is mainly resulted from the integrator adopted in the APC.

The magnitude analysis also provides an overall understanding on how the control loops affect the VSC impedance in a wide frequency range, which can be helpful to understand their impacts on stability: the magnitude analysis can be compared with the system oscillation modes to analyze the sensitivity of different control loops to stability. For instance, according to the analysis in Section III, the system oscillation modes, which should be close to the phase crossover frequencies in critically unstable conditions, are found around $46.6 \mathrm{~Hz}$ and $53.4 \mathrm{~Hz}$. The two frequencies locate within the red dashed ellipses in Fig. 11, thus the stability studied in this case is sensitive to both VC and APC parameters. Since the RPC's impact is weaker, the stability should be less sensitive to the RPC parameters.

It is noted that the above results are merely carried out based on the droop-controlled VSC. If the grid-forming VSC adopts different control schemes or different types of controllers, e.g., if an integrator is adopted in the RPC, then the RPC's impact around the fundament frequency is not negligible compared with the APC. Although the results may vary from cases to cases, such comparative analysis approach can still be applied to gain more insights into the control-loop impacts for a design-oriented study.

\section{Design-Oriented Controller Tuning}

After knowing the control-loop impacts on the VSC
Table III. Study Cases Considering Parameter Changing

\begin{tabular}{c|c|c|c|c}
\hline \hline Cases & SCR & VC BW & APC $\boldsymbol{m}_{\boldsymbol{p}}$ & $\mathbf{R P C} \boldsymbol{n}_{\boldsymbol{q}}$ \\
\hline $\mathrm{A}$ & 10 & $57 \mathrm{~Hz}$ & $2 \% \omega_{0} / P_{0}$ & $10 \% V_{\mathrm{ref}} / Q_{0}$ \\
$\mathrm{~B}$ & 7 & $57 \mathrm{~Hz}$ & $2 \% \omega_{0} / P_{0}$ & $10 \% V_{\mathrm{ref}} / Q_{0}$ \\
$\mathrm{C}$ & 10 & $167 \mathrm{~Hz}$ & $2 \% \omega_{0} / P_{0}$ & $10 \% V_{\mathrm{ref}} / Q_{0}$ \\
$\mathrm{D}$ & 10 & $57 \mathrm{~Hz}$ & $1 \% \omega_{0} / P_{0}$ & $10 \% V_{\mathrm{ref}} / Q_{0}$ \\
$\mathrm{E}$ & 10 & $57 \mathrm{~Hz}$ & $2 \% \omega_{0} / P_{0}$ & $1 \% V_{\mathrm{ref}} / Q_{0}$ \\
\hline \hline
\end{tabular}

impedance, a design-oriented controller design can be conducted for stability enhancement: the controller tuning can be prioritized on the sensitive control loops.

Cases C-E are analyzed, respectively, to investigate the impacts of the VC, the APC, and the RPC on the stability. The key changing parameters compared with Case A are listed in Table III. The stability analysis with the generalized NSC is shown in Fig. 12, which can be compared with the analysis result for Case A shown in Fig. 6(b). It is seen from Fig. 12(a)-(b) that by increasing the VC bandwidth or decreasing the APC droop coefficient, the system stability can be effectively improved. This supports the previous control-loop impact analysis, that the VC and APC are both sensitive to the stability. Such control parameter tuning effects can be clarified from the small-gain perspective: since increasing the VC bandwidth and reducing the APC droop coefficient both reduce the impedance magnitudes of $\mathbf{Z}_{\mathrm{o}}$ and $\mathbf{Z}_{\text {refP }}$ significantly around the fundamental frequency, the VSC impedance magnitude can thus be reduced accordingly. The singular value analysis in Section III has indicated that the high VSC magnitude is the root cause of instability of grid-forming VSCs in stiff grids in the VSC non-passive region. Therefore, reducing the VSC impedance magnitude can improve the system stability. However, it should be noted that the smallgain principle for the parameter tuning is only a sufficient condition. It may fail in some cases if the phase response is influenced more significantly, e.g., if second-order poles or zeros exist around the oscillation modes, then an accurate stability analysis with the NSC is needed. As for the RPC loop, reducing the RPC droop coefficient even for 10 times, the system still maintains in a critically unstable condition, as shown in Fig. 12(c). It proves that the RPC is not sensitive to the stability.

A flowchart can thus be summarized in Fig. 13 for a designoriented control design based on the impedance decomposition. It is noted that the approach gains more insights into the control-loop impacts of grid-forming VSCs, which can be also applied to VSCs with other schemes of grid-forming control. The analysis can provide some guidance for the controller tuning, yet the accurate stability analysis still relies on the entire system model.

Furthermore, the impedance decomposition approach is not limited to grid-forming VSCs. In duality, grid-following VSCs can be decomposed into several admittances in parallel, which has been introduced in [47] to analyze the control-loop impacts on the VSC passivity. 

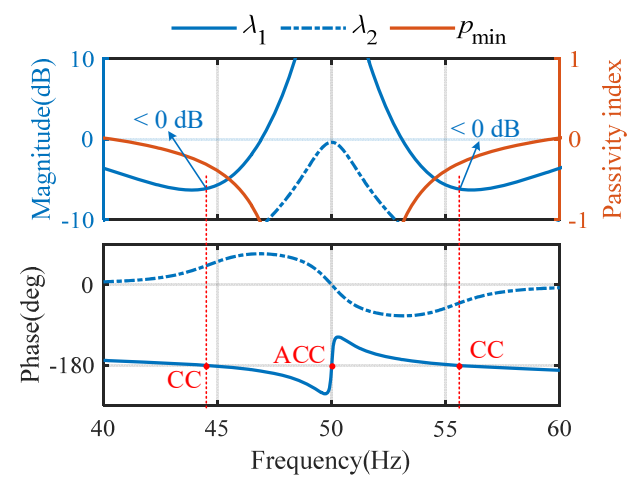

(a)

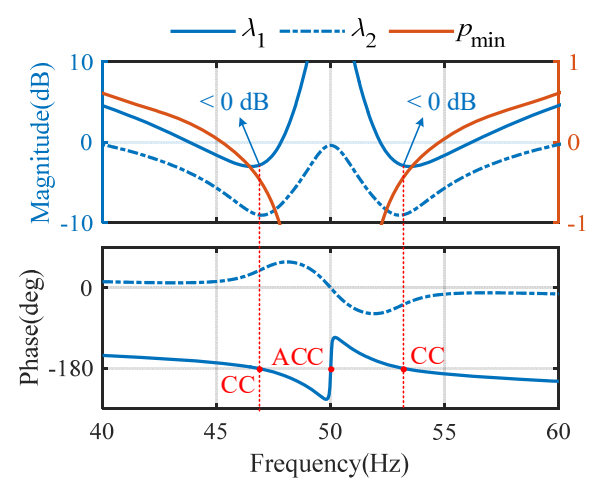

(b)
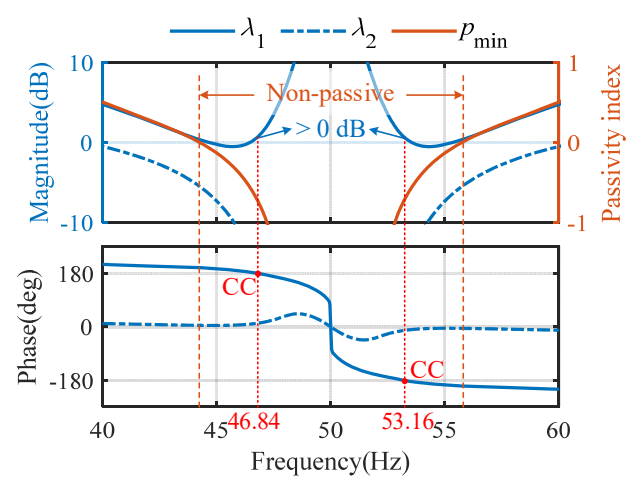

(c)

Fig. 12 Stability analysis by controller tuning for Cases C-E. (a) Case C: increasing VC bandwidth; (b) Case D: decreasing APC droop coefficient; (c) Case E: decreasing RPC droop coefficient.

\section{CASE VALidATIONS}

This section validates the previous stability analysis of Cases A-E by experiments or simulations.

Fig. 14 shows the experimental setup. A grid simulator (Chroma 61845) is used to generate an ideal three-phase voltage source. The VSC is fed by a DC source and the control is implemented in dSPACE (DS1007). The circuit parameters and the VSC control parameters are the same as given in Tables I-II. Two grid scenarios with different short-circuit

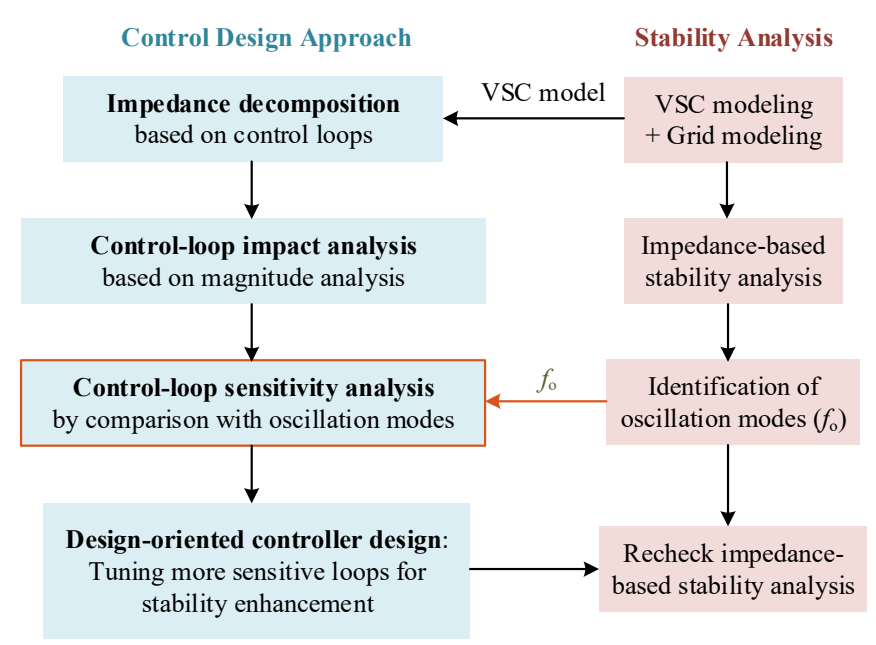

Fig. 13 Flowchart of the passivity-based controller design approach based on impedance decomposition.

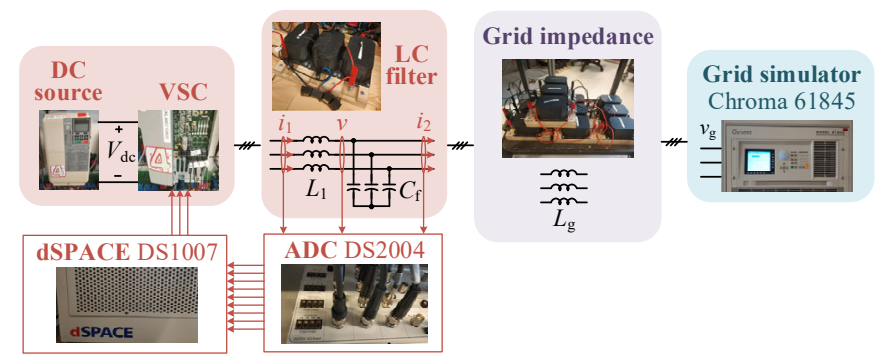

Fig. 14 Experimental setup of the grid-connected grid-forming VSC.

ratios (SCRs) defined at the $\mathrm{PoC}$ are considered, i.e., $L_{\mathrm{g}}=6$ $\mathrm{mH}(\mathrm{SCR} \approx 10)$ and $L_{\mathrm{g}}=8 \mathrm{mH}(\mathrm{SCR} \approx 7)$.

The experimental waveforms for Case A are provided in Fig. 15. It can be found that the system is critically unstable with sideband oscillations, which verifies the stability analysis shown in Fig. 6. Moreover, the discrete Fourier transformation (DFT) analysis identifies the oscillation frequency of $3.3 \mathrm{~Hz}$ in the power waveforms, which turns out to be $46.7 \mathrm{~Hz}$ and 53.3 $\mathrm{Hz}$ in the $\alpha \beta$ frame. The sideband oscillation frequencies are also well predicted by the analysis result in Fig. 6 . Therefore, the proposed impedance model of the VSC can accurately analyze the system stability.

The experimental waveforms for Case B when the grid becomes weaker are provided in Fig. 16. Compared with Fig. 15 , the system can be stabilized, which verifies the analysis in Fig. 7.

The impacts of VC bandwidth, APC droop coefficient, and RPC droop coefficient are further studied by Cases C-E, and the corresponding waveforms are given in Figs. 17-19, respectively. Fig. 17 verifies that the increase of the VC loop bandwidth can enhance the stability, which agrees with the analysis in Fig. 12(a). Fig. 18 indicates that the decrease of the APC droop coefficient also stabilizes the system, which agrees with the analysis in Fig. 12(b). However, in Fig. 9, the system still maintains in a critically unstable condition, with the RPC droop coefficient decreased. This also verifies the stability analysis in Fig. 12(c). 
IEEE TechRxiv

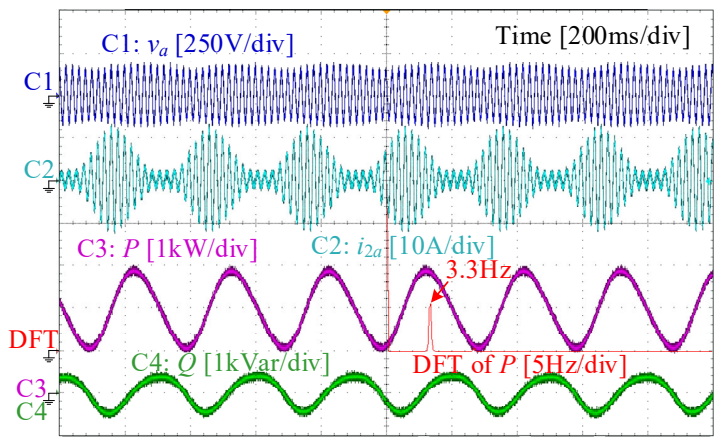

Fig. 15 Experimental waveforms for Case A.

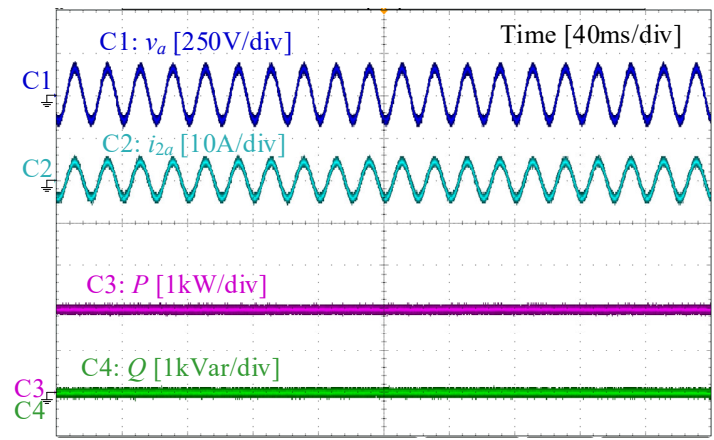

Fig. 16 Experimental waveforms for Case B with $L_{\mathrm{g}}$ increased (weaker grid).

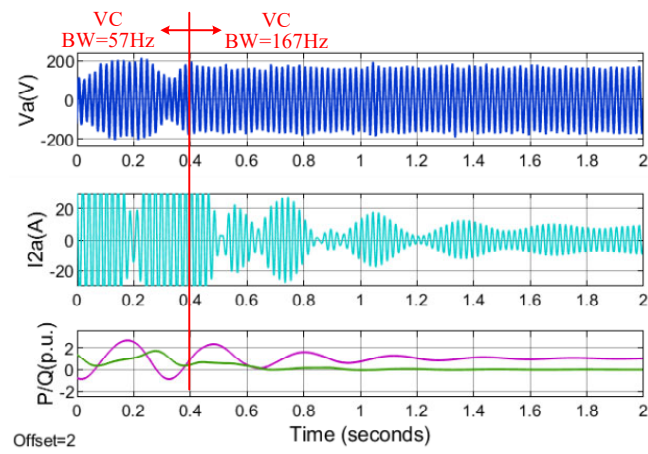

Fig. 17 Simulation waveforms for Case $\mathrm{C}$ with VC bandwidth increased.

\section{CONCLUSION}

The complex-valued $\alpha \beta$-frame impedance model of the droop-controlled grid-forming VSC has been established in this paper to investigate the stability in stiff grids. Based on the impedance decomposition approach, the VC, APC and RPC's impacts have been decomposed into three impedance terms in series. Through the magnitude analysis of the decomposed impedances, it has been found that for the droopcontrolled VSC:

1) The integrator used in the APC results in high magnitude in the VSC output impedance around the fundamental frequency, which tends to result in the instability in stiff grids.

2) The VC and APC is more sensitive to the instability than the RPC.

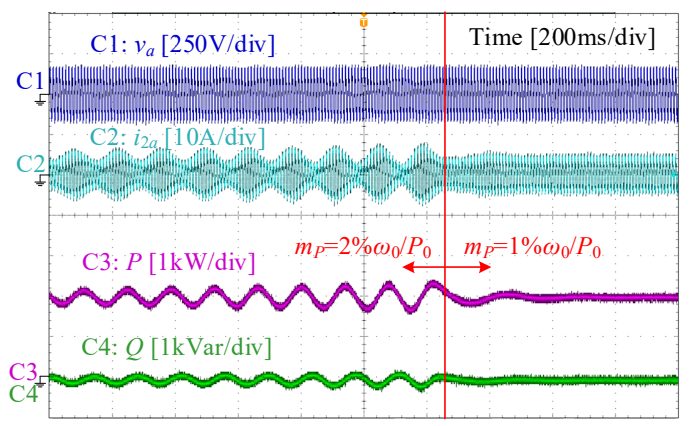

Fig. 18 Experimental waveforms for Case D with APC droop control coefficient reduced.

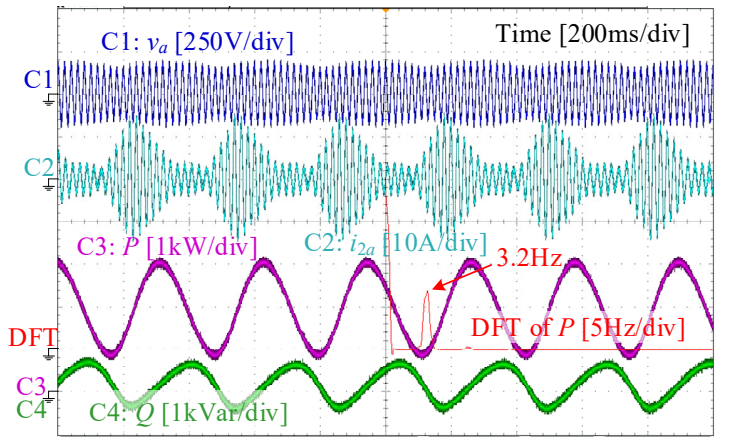

Fig. 19 Experimental waveforms for Case F with RPC droop control coefficient reduced.

Based on the above findings, the design-oriented controller design by tuning VC and APC parameters in priority has been implemented for stability enhancement, which has been validated by the stability analysis, simulations and experiments.

\section{REFERENCES}

[1] B. Kroposki, B. Johnson, Y. Zhang, V. Gevorgian, P. Denholm, B. Hodge, and B. Hannegan, "Achieving a 100\% renewable grid: operating electric power systems with extremely high Levels of variable renewable energy," IEEE Power and Energy Mag., vol. 15, no. 2, pp. 61-73, Mar. - Apr. 2017.

[2] R. H. Lasseter, Z. Chen, and D. Pattabiraman, "Grid-forming Inverters: a critical asset for the power grid," IEEE J. Emerging Sel. Topics Power Electron., vol. 8, no. 2, pp. 925-935, Jun. 2020.

[3] X. Wang and F. Blaabjerg, "Harmonic Stability in Power Electronic Based Power Systems: Concept, Modeling, and Analysis," IEEE Trans. Smart Grid, vol. 10, no. 3, pp. 2858-2870, May 2019.

[4] J. Z. Zhou, H. Ding, S. Fan, Y. Zhang and A. M. Gole, "Impact of ShortCircuit Ratio and Phase-Locked-Loop Parameters on the Small-Signal Behavior of a VSC-HVDC Converter," IEEE Trans. Power Del., vol. 29, no. 5, pp. 2287-2296, Oct. 2014.

[5] H. Liu, X. Xie, J. He, and et al., "Subsynchronous interaction between direct-drive PMSG based wind farms and weak AC networks," IEEE Trans. Power Syst., vol. 32, no. 6, pp. 4708-4720, Nov. 2017.

[6] IEEE subsynchronous resonance working group, "Terms, Definitions and Symbols for Subsynchronous Oscillations," IEEE Trans. Power Apparat. and Syst., vol. PAS-104, no. 6, pp. 1326-1334, Jun. 1985.

[7] L. Harnefors, M. Bongiorno and S. Lundberg, "Input-admittance calculation and shaping for controlled voltage-source converters," IEEE Trans. Ind. Electron., vol. 54, no. 6, pp. 3323-3334, Dec. 2007.

[8] B. Wen, D. Boroyevich, R. Burgos, P. Mattavelli, Z. Shen, "Analysis of D-Q small-signal impedance of grid-tied inverters," IEEE Trans. Power Electron., vol. 31, no. 1, pp. 675-687, Jan. 2016. 


\section{IEEE TechRxiv}

[9] B. Wen, D. Dong, D. Boroyevich, R. Burgos, P. Mattavelli, and Z. Shen, "Impedance-based analysis of grid-synchronization stability for threephase paralleled converters," IEEE Trans. Power Electron., vol. 31, no. 1, pp. 26-38, Jan. 2016.

[10] X. Wang, L. Harnefors, and F. Blaabjerg, "Unified impedance model of grid-connected voltage-source converters," IEEE Trans. Power Electron., vol. 33, no. 2, pp. 1775-1787, Feb. 2018.

[11] H. Laaksonen, P. Saari, and R. Komulainen, "Voltage and frequency control of inverter based weak LV network microgrid," in Proc. Inter. Conf. on Future Power Syst., Amsterdam, Netherlands, Nov. 2005.

[12] L. Zhang, L. Harnefors, and H. Nee, "Power-synchronization control of grid-connected voltage-source converters," IEEE Trans. Power Syst., vol. 25 , no. 2, pp. 809-820, May 2010.

[13] H. Wu and X. Wang, "Design-oriented transient stability analysis of grid-connected converters with power synchronization control," IEEE Trans. Ind. Electron., vol. 66, no. 8, pp. 6473-6482, Aug. 2019.

[14] D. Pan, X. Wang, F. Liu, and R. Shi, "Transient stability of voltagesource converters with grid-forming control: a design-oriented study," IEEE Journ. of Emerg. and Select. Topics in Power Electron., Early Access, DOI: 10.1109/JESTPE.2019.2946310.

[15] J. Rocabert, A. Luna, F. Blaabjerg, and P. Rodriguez, "Control of power converters in AC microgrids," IEEE Trans. Power Electron., vol. 27, no. 11, pp. 4734-4749, Nov. 2012.

[16] L. Zhang, L. Harnefors, and H. Nee, "Interconnection of two very weak AC systems by VSC-HVDC links using power-synchronization control," IEEE Trans. Power Syst., vol. 26, no. 1, pp. 344-355, Feb. 2011.

[17] P. Mitra, L. Zhang, and L. Harnefors, "Offshore wind integration to a weak grid by VSC-HVDC links using power-synchronization control: a case study," IEEE Trans. Power Del., vol. 29, no. 1, pp. 453-461, Feb. 2014.

[18] J. Khazaei, Z. Miao, and L. Piyasinghe, "Impedance-model-based MIMO analysis of power synchronization control," Electric Power Systems Research, vol. 154, pp. 341-351, Jan. 2018.

[19] L. Huang, H. Xin, and Z. Wang, "Damping low-frequency oscillations through VSC-HVDC stations operated as virtual synchronous machines," IEEE Trans. Power Electron., vol. 34, no. 6, pp. 5803-5818, Jun. 2019.

[20] G. Denis, T. Prevost, P. Panciatici, X. Kestelyn, F. Colas, and X. Guillaud, "Improving robustness against grid stiffness, with internal control of an AC voltage-controlled VSC.," in Proc. IEEE Power and Energy Society General Meeting (PESGM), Boston, MA, Nov. 2016.

[21] T. Qoria, F. Gruson, F. Colas, X. Kestelyn, X. Guillaud, "Analysis of the coupling between the outer and inner control loops of a Grid-forming Voltage Source Converter," in Proc. Europ. Conf. Power Electron. and Appl. (EPE), Sep. 2020, preprint.

[22] S. D'Arco, J. A. Suul, and O. Fosso, "Automatic tuning of cascaded controllers for power converters using eigenvalue parametric sensitivities," IEEE Trans. Ind. Appl., vol. 51, no. 2, pp. 1743-1753, Mar. - Apr. 2015.

[23] E. Barklund, N. Pogaku, M. Prodanovic, C. H.-Aramburo, and T. C. Green, "Energy management in autonomous microgrid using stabilityconstrained droop control of inverters," IEEE Trans. Power Electron., vol. 23, no. 5, pp. 2346-2352, Sep. 2008.

[24] K. Yu, Q. Ai, S. Wang, J. Ni, and T. Lv, "Analysis and optimization of droop controller for microgrid system based on small-signal dynamic model," IEEE Trans. Smart Grid, vol. 7 no. 2, pp. 695-705, Mar. 2016.

[25] Y. Prabowo, V. M. Iyer, B. Kim, and S. Bhattacharya, "Modeling and stability assessment of single-phase droop controlled solid state transformer," in Proc. Inter. Conf. on Power Electron. and ECCE Asia, Busan, Korea, May 2019, pp. 3285-3291.

[26] X. Wang, F. Blaabjerg, and W. Wu, "Modeling and analysis of harmonic stability in an AC power-electronics-based power system," IEEE Trans. Power Electron., vol. 29, no. 12, pp. 6421-6432, Dec. 2014.

[27] L. Harnefors, X. Wang, A. G. Yepes, and F. Blaabjerg, "Passivity based stability assessment of grid-connected VSCs - an overview," IEEE J. Emerging Sel. Topics Power Electron., vol. 4, no. 1, pp. 116-125, Mar. 2016.

[28] G. Denis, "From grid-following to grid-forming: The new strategy to build $100 \%$ power electronics interfaced transmission system with enhanced transient behavior," Ph.D. dissertation, Energie électrique, Ecole Centrale de Lille, France, 2017.

[29] S. Wang, Z. Liu, J. Liu, D. Boroyevich, and R. Burgos, "Small-signal modeling and stability prediction of parallel droop-controlled inverters based on terminal characteristics of individual inverters," IEEE Trans. Power Electron., Early Access, DOI: 10.1109/TPEL.2019.2914176.

[30] W. Cao, Y. Ma, F. Wang, L. M. Tolbert, and Y. Xue, "Low-frequency stability analysis of inverter-based islanded multiple-bus AC microgrids based on terminal characteristics", IEEE Trans. Smart Grid, Early Access, DOI: 10.1109/TSG.2020.2978250.

[31] F. Cavazzana, A. Khodamoradi, H. Abedini, and P. Mattavelli, "Analysis of an impedance modeling approach for droop-controlled inverters in system DQ frame," in Proc. IEEE Energy Conversion Congress and Exposition (ECCE), Baltimore, MD, USA, Sep. 2019.

[32] Y. Liao, X. Wang, F. Liu, K. Xin and Y. Liu, "Sub-Synchronous Control Interaction in Grid-Forming VSCs with Droop Control," in Proc. IEEE Workshop on the Electronic Grid (eGRID), Xiamen, China, Nov. 2019, pp. 1-6.

[33] K. Oue, S. Sano, T. Kato, and K. Inoue, "Stability analysis of gridforming inverter in DQ frequency domain," in Proc. IEEE Workshop on Control and Modeling for Power Electronics (COMPEL), Toronto, ON, Canada, Jun. 2019.

[34] S. D'Arco and J. A. Suul, "Equivalence of virtual synchronous machines and frequency- droops for converter-based microgrids," IEEE Trans. Smart Grid, vol. 5, no. 1, pp. 394-395, Jan. 2014.

[35] P. C. Loh, M. J. Newman, D. N. Zmood, and D. G. Holmes, "A comparative analysis of multiloop voltage regulation strategies for single and three-Phase UPS systems," IEEE Trans. Power Electron., vol. 18, no. 5, pp. 1176-1185, Sep. 2003.

[36] X. Wang, P. C. Loh, and F. Blaabjerg, "Stability analysis and controller synthesis for single-loop voltage-controlled VSIs," IEEE Trans. Power Electron., vol. 32, no. 9, pp. 7394-7404, Sep. 2017.

[37] Y. Liao, X. Wang and F. Blaabjerg, "Passivity-Based Analysis and Design of Linear Voltage Controllers For Voltage-Source Converters," IEEE O. J. Ind. Electron. Soc., vol. 1, pp. 114-126, 2020.

[38] Y. W. Li, "Control and resonance damping of voltage-source and current source converters with LC filters," IEEE Trans. Ind. Electron., vol. 56, no. 5, pp. 1511-1521, May 2009.

[39] J. Ma, X. Wang, F. Blaabjerg, L. Harnefors, and W. Song, “Accuracy analysis of the zero-order hold model for digital pulse width modulation," IEEE Trans. Power Electron., vol. 33, no. 12, pp. 1082610834, Dec. 2018.

[40] Y. Liao and X. Wang, "Stationary-frame complex-valued frequencydomain modeling of three-phase power converters," IEEE J. Emerg. Sel. Topics Power Electron., vol. 8, no. 2, pp. 1922-1933, Jun. 2020.

[41] Z. Shen, "Online measurement of three-phase AC power system impedance in synchronous coordinates," Ph.D. dissertation, Dept. of Elect. Eng., Virginia Tech, Blacksburg, USA, 2012.

[42] J. Bao, F. Wang, P. L. Lee, and W. Zhou, "New frequency-domain phase-related properties of MIMO LTI passive systems and robust controller synthesis," in Proc. 13th World Congr of IFAC, San Francisco, USA, Jun.-Jul. 1996, pp. 3276-3281.

[43] A. J. Agbemuko, J. L. Dominguez-Garcia, O. Gomis-Bellmunt, and L. Harnefors, "Passivity-based analysis and performance enhancement of a vector controlled VSC connected to a weak ac grid," IEEE Trans. Power Delivery, Early Access, DOI: 10.1109/TPWRD.2020.2982498.

[44] I. Postlethwaite, "A generalized inverse Nyquist stability criterion," Int. J. Control, vol. 26, no. 3, pp. 325-340, 1977.

[45] Y. Liao and X. Wang, "Impedance-based stability analysis for interconnected converter systems with open-loop RHP poles," IEEE Trans. Power Electron., vol. 35, no. 4, pp. 4388-4397, Apr. 2020.

[46] Y. Geng, Y. Yun, R. Chen, K. Wang, H. Bai, and X. Wu, "Parameters design and optimization for LC-type off-grid inverters with inductorcurrent feedback active damping," IEEE Trans. Power Electron., vol. 33, no. 1, pp. 703-715, Jan. 2018.

[47] L. Harnefors, X. Wang, S. Chou, M. Bongiorno, M. Hinkkanen and M. Routimo, "Asymmetric Complex-Vector Models With Application to VSC-Grid Interaction," IEEE J. Emerg. Sel. Topics Power Electron., vol. 8, no. 2, pp. 1911-1921, Jun. 2020. 